\title{
Pediatric Ophthalmology and Strabismus in Italy
}

Paolo Nucci, MD

$\mathrm{T}$ he most famous Italian strabismologist of the $20^{\text {th }}$ century is the recently retired Bruno Bagolini. Decades before him, his mentor Bietti had suggested surgical procedures for congenital nystagmus similar to those recently popularized in the United States. Neither pediatric ophthalmology nor strabismus (PO\&S) are officially recognized as subspecialties in Italy, although throughout Italy one finds ophthalmologists styled as specialists in pediatric ophthalmology and strabismus. During the last 20 years, several departments dedicated to PO\&S have been established in some universities and hospitals (especially children's hospitals), but there is no official recognition of any specialized training in PO\&S. Traditionally, centers of excellence in PO\&S have existed in Milan, Como, Trieste, Genova, Modena, Bologna, Florence, Siena, Rome, Bari, Messina, and Naples.

A few years ago, Italy restructured the financing for its health care system. Previously, all resources came from national funds. Now each region is responsible for financing and organizing its own health care. This lead to regional differences in reimbursement. Pediatric eye care is not prioritized highly. Rather, it is generally considered to be time consuming and is usually poorly remunerated.

Of a total population of 60 million, there are 10 million children $>12$ years. Their primary care is provided by $>20,000$ pediatricians. There are 7,000 ophthalmologists, most of whom are members of the Italian Society of Ophthalmology (SOI). The society sponsors subspecialty sections, among them PO\&S. Meetings take place twice a year, usually with invited lectures on PO\&S topics and presentations of case reports or unpublished series. Fewer than 300 ophthalmologists have a declared interest in PO\&S, eg, affiliation with one of the national (eg, Società Italiana di Oftalmologia Pediatrica [SIOP] or Associazione Italiana Strabismo [AIS]) or international (eg, American Association for Pediatric Ophthalmology and Strabismus [AAPOS], European Strabismological Association [ESA], International Strabismological Association [ISA]), PO\&S

From the Department of Ophthalmology, Pediatric Opbthalmology Unit, San Paolo Hospital, University of Milan, Milan Italy

Submitted October 3, 2003.

Revision accepted October 10, 2003.

Reprint requests: Paolo Nucci, MD, Pediatric Ophthalmology Unit, San Paolo Hospital,

Viale E Caldara 7, Milan 20090, Italy

7 AAPOS 2004;8:220-221

Copyright (C) 2004 by the American Association for Pediatric Ophthalmology and Strabismus.

1091-8531/2004/\$35.00+0

doi:10.1016/j.jaapos.2003.10.004 societies. Of these 300 or so ophthalmologists, it is not known how many have complete fellowships overseas; I estimate $<20$, and most of these practice in northern and central Italy. There are many trained orthoptists in Italy, but, unfortunately, many are unemployed because hospitals prefer to employ nurses to do orthoptic work even though they do not have the appropriate training.

After students complete secondary school at age 19 years or so, each university offers a competitive entrance examination to their medical school. There is a 6-year undergraduate course with a syllabus that is uniform across the country. The degree of Doctor in Medicine and Surgery is awarded after many examinations as well as presentation of a thesis by dissertation on specific and modern aspects of medicine. Usually the candidates prepare a thesis on a topic consistent with his or her residency choice. Entrance to postgraduate training is by way of a highly competitive examination, and the candidate must first obtain a state medical license to practice in the country.

The ophthalmology residency program lasts 4 years. It is supervised by a university department and takes place in a university or teaching hospital. The syllabus is similar to that in other European countries. In each of the 4 years, there is an examination that includes evaluation of theoretical knowledge and clinical skills. Within the European community there is supposed to be equivalency of standards and training. Italian medicine does not have a tradition of overseas fellowships, although there have been exceptions in the past 15 years.

Private consulting and surgical ophthalmology practice is largely confined to general ophthalmology or refractive and cataract surgeons. University- and hospital-based practice is poorly paid; a typical (insulting) stipend is 25,000 to $45,000 €$ per year. This can sometimes be supplemented by private work under the institutional "flag" (the income goes to the institution, which returns a variable percentage to the doctor).

At this time, no fellowship, master, or other academic courses are available in Italy for training as a PO\&S specialist. To become a PO\&S "specialist," an ophthalmologist usually attends a PO\&S unit for a certain period and then starts practicing in the field without any special requirements.

The prevalence of ocular conditions in Italy is quite similar to that in the United States. Vernal conjunctivitis may have a higher prevalence, whereas infectious diseases are relatively uncommon. Unfortunately, epidemiologic observations are rarely carried out by the Italian health 
care system, and single institution is often unable to collect unbiased data.

All of the aspects of PO\&S, including genetics, are currently studied in Italy, and cooperation with foreign institutions is strongly pursued. Amblyopia studies and refractive surgery in children and strabismus patients are the most recent fields of interest. Among non-English speaking countries, Italian efforts to publish in Englishlanguage journals are very strong. Ophthalmology, American Fournal of Ophthalmology, Fournal of Cataract and Refractive Surgery, European Fournal of Ophthalmology (whose editor and publishing company are Italian) and Investigative Ophthalmology \& Vision Science (IOVS) are the preferred journals.

We have increasingly moved to the family physician as gatekeeper in our medical system. As a result, more referrals are being made to general ophthalmologists regardless of the complexity of the patient's problem. One result is less-efficient and less-effective treatment for pediatric eye problems. A second outcome is lessened interest in young ophthalmologists pursuing subspecialist training.

I look forward having to a fully trained PO\&S specialist in every large city hospital and university Department of Ophthalmology. Infants and children are not merely little adults, and optimal pediatric care requires a thorough understanding of the unique medical and surgical problems encountered in these patients. Furthermore, Italy needs to invest the health budget more wisely to better implement new tools and techniques because as too many politicians consider health care to be a bottomless pit without sufficient regard to the quality of service provided.

$I$ am indebted to Marilyn B. Mets and Albert $W$. Biglan for introducing me to the field of pediatric ophthalmology and strabismus in the United States. I also thank Adriano Magli (AIS president), Riccardo Frosini (AIS past president), Benedetto Ricci (SIOP president), and Paolo E. Bianchi (SIOP vice president) for sharing their ideas on this topic with me. 